\title{
Comparison of the Prognostic Value of Global and Regional Myocardial Flow Capacity Radius, Myocardial Flow Reserve, and Stress Myocardial Blood Flow Using Rubidium-82 with SiPM PET/CT
}

\section{Matthieu DIETZ ( $\sim$ Matthieu.dietz@chuv.ch )}

CHUV: Centre Hospitalier Universitaire Vaudois https://orcid.org/0000-0001-6041-2531

\section{Christel H Kamani}

CHUV: Centre Hospitalier Universitaire Vaudois

Gilles Allenbach

CHUV: Centre Hospitalier Universitaire Vaudois

Vladimir Rubimbura

CHUV: Centre Hospitalier Universitaire Vaudois

\section{Stephane Fournier}

CHUV: Centre Hospitalier Universitaire Vaudois

\section{Marie Nicod Lalonde}

CHUV: Centre Hospitalier Universitaire Vaudois

\section{Niklaus Schaefer}

CHUV: Centre Hospitalier Universitaire Vaudois

\section{Eric Eeckhout}

CHUV: Centre Hospitalier Universitaire Vaudois

Olivier Muller

CHUV: Centre Hospitalier Universitaire Vaudois

John $O$ Prior

CHUV: Centre Hospitalier Universitaire Vaudois https://orcid.org/0000-0003-1429-1374

\section{Research Article}

Keywords: cardiac positron emission tomography, quantitative myocardial perfusion, major adverse cardiovascular events, outcome

Posted Date: December 2nd, 2021

DOI: https://doi.org/10.21203/rs.3.rs-1122028/v1 
License: (c) (i) This work is licensed under a Creative Commons Attribution 4.0 International License. Read Full License 


\section{Abstract \\ Purpose}

The aim of this study was to assess the most reliable quantitative variable on Rubidium-82 $\left({ }^{82} \mathrm{Rb}\right)$ cardiac PET/CT for predicting major adverse cardiovascular events (MACE), on the latest PET camera using silicon photomultipliers digital readout (SiPM) technology.

\section{Methods}

We prospectively enrolled 274 consecutive participants with suspected myocardial ischemia. Participants underwent ${ }^{82} \mathrm{Rb}$ cardiac SiPM PET/CT and were followed-up for MACE over 652 days (interquartile range: 559 to 751 days). For each participant, global and regional myocardial flow reserve (MFR), stress myocardial blood flow (stress MBF) and their combination as myocardial flow capacity radius (MFC radius) were measured.

\section{Results}

On receiver operator curve analysis, MACE prediction was similar for global and regional MFR, stress MBF, and MFC radius (area under the curve; (i) Global: 0.70 vs. 0.71 and 0.73 , and (ii) Regional: 0.71 vs. 0.71 and 0.73 , respectively, $p>0.1$ for all pairwise comparisons). On multivariable analysis, (i) Global: MFR < 1.98, stress MBF $<1.94 \mathrm{~mL} / \mathrm{g} / \mathrm{min}$, and MFC radius $<3.12$, as well as (ii) Regional: $M F R<1.75$, stress $\mathrm{MBF}<1.75 \mathrm{~mL} / \mathrm{g} / \mathrm{min}$, and MFC radius $<2.7$, emerged all as similar independent predictors of $\operatorname{MACE}(p<0.001$ for all $)$.

\section{Conclusions}

Using the latest SiPM PET technology with ${ }^{82} \mathrm{Rb}$, global and regional MFR, stress MBF, and MFC radius are similar powerful predictors of cardiovascular event.

\section{Introduction}

Myocardial perfusion imaging is a powerful non-invasive functional tool for risk stratification, recommended by clinical practice guidelines $(1-5)$. Compared with relative perfusion images, absolute quantification of myocardial blood flow (MBF) and myocardial flow reserve (MFR) by positron emission tomography (PET) could improve risk stratification (6). Global and regional perfusion provide information on different aspects of myocardial perfusion. Impairment in global perfusion may be caused by either multivessel epicardial disease or microcirculatory dysfunction. Regional absolute perfusion 
measurements may enable the additive detection of small regional defects caused by epicardial coronary artery disease (CAD), which could not be detected with average global perfusion measurements (6).

The myocardial flow capacity (MFC) concept is an alternative approach integrating both MFR and stress MBF to depict MBF $(6,7)$. MFC may overcome some of the limitations of using MFR or stress MBF alone and represents a promising tool to improve clinical decision-making (7-9). However, despite a robust conceptual validation and recently promising clinical data especially after revascularization, studies demonstrating added clinical value of the MFC concept over stress MBF and MFR are still limited (8-10).

Silicon photomultipliers with digital readout (SiPM) PET is a new PET technology with improved spatial and timing resolution and a relatively high sensitivity and count-rate capability as compared to PET scanners using conventional photomultiplier tubes (11). Rubidium-82 $\left({ }^{82} \mathrm{Rb}\right)$ is a widely clinically used PET perfusion tracer produced from a strontium-82 $\left({ }^{82} \mathrm{Sr}\right) /{ }^{82} \mathrm{Rb}$ generator.

The most reliable quantitative variable on ${ }^{82} \mathrm{Rb}$ cardiac $\mathrm{PET} / \mathrm{CT}$ for predicting cardiovascular events is not fully established and is unknown using SiPM technology.

The aim of this study was to prospectively compare on a SiPM PET camera, with ${ }^{82} \mathrm{Rb}$, the prognostic value for cardiovascular events of global and regional MFR, stress MBF, and MFC radius.

\section{Methods}

\section{Study Population}

We prospectively enrolled participants with suspected myocardial ischemia to undergo ${ }^{82} \mathrm{Rb}$ cardiac SiPM PET/CT between June 2018 and June 2019 at the Lausanne University Hospital. Participants' cardiovascular risk factors and medication use were ascertained at time of PET imaging. The Local Ethics Committee approved this study protocol (\#PB_2017-00634), and all participants gave written informed consent prior to inclusion.

\section{Imaging protocol with SiPM ${ }^{82} \mathrm{Rb} \mathrm{PET} / \mathrm{CT}$}

For each participant, a rest and adenosine or regadenoson stress SiPM PET/CT scan was performed, using a single dedicated camera (Biograph Vision 600, Siemens Medical Solutions, Knoxville, USA). Participants were instructed to fast for $6 \mathrm{~h}$ and avoid caffeine-containing food or beverages $24 \mathrm{~h}$ prior to the test. At rest, a $15-25$ s i.v. infusion of $5 \mathrm{MBq} / \mathrm{Kg}$ of ${ }^{82} \mathrm{Rb}$ (Ruby-Fill ${ }^{\circledR}$ generator and $\mathrm{Rb}-82$ elution system [v3], Jubilant DraxImage, Kirkland, QC, Canada) was administered with an automatic infusion system and 3D dynamic PET images were acquired starting at the beginning of the infusion over 6 min $19 \mathrm{~s}(12 \times 8,5 \times 12,1 \times 30,1 \times 60$, and $1 \times 120 \mathrm{~s})$. A second acquisition was then started following the same protocol with similar activity 2 min into an adenosine infusion $(140 \mathrm{mg} / \mathrm{kg} / \mathrm{min}$ over $6 \mathrm{~min})$ or following a regadenoson administration (400 $\mu$ g over $10 \mathrm{~s})$. A low-dose CT (100 keV, 16 mAs) transmission scan was used for attenuation correction. Images were reconstructed by ordered subsets 
expectation maximization algorithms (4 iterations, 5 subsets, $4.0 \mathrm{~mm}$ FWHM gaussian post-filter, $220 \times$ 220-pixel matrix size). Blood pressure, heart rate, and a 12-lead ECG were recorded throughout the procedure. The radiation dose for a $70 \mathrm{~kg}$ participant was estimated to be $2 \times 0.39 \mathrm{mSv}$ for rest and stress ${ }^{82} \mathrm{Rb}$, and $1 \times 0.17 \mathrm{mSv}$ for the low-dose attenuation correction CT plus CT scout, resulting in a total dose of $0.95 \mathrm{mSv}$.

\section{Usual quantitative myocardial perfusion analysis}

Perfusion was assessed quantitatively measuring MBF in milliliter per gram per minute at rest and stress, using the highly automated FlowQuant v2.7 software (Ottawa, Ontario, Canada), with a 1-tissue compartment model with a flow-dependent extraction correction (12). MFR was calculated; MFR = stress $\mathrm{MBF} /$ rest MBF. Rate-pressure product adjusted rest MBF and MFR were determined to account for high resting heart rate or systolic blood pressure by multiplying rest MBF by $8500 \mathrm{mmHg} / \mathrm{min}$ and dividing by rate-pressure product (resting heart rate multiplied by resting systolic blood pressure). To reduce the potential spillover in image-derived blood activity curves, a dual spill-over correction was systematically applied (13), as well as global partial-volume recovery correction and motion correction (14).

\section{Myocardial flow capacity}

MFC, developed by Johnson and Gould using ${ }^{82} \mathrm{Rb} P E T$ imaging, is a metric that integrates combinations of resting MBF, stress MBF and MFR (7). To select an optimal threshold value for MFC and allow a straightforward and reliable comparison between MFC, MFR and stress MBF, the Euclidian distance in the Gould MFC diagram ( $x=$ stress MBF, $y=$ MFR) was calculated to stratify MFC as per patient single continuous variables. This newly developed variable was called "MFC radius" and defined as MFC radius $=\sqrt{(\text { stress } M B F)^{2}+M F R^{2}}$.

\section{Quantitative analysis}

In the current study we used continuous variables expressed as the average MFR and stress MBF values from (i) the entire ventricle (global MFR, global stress MBF and global MFC) and (ii) all vascular territories derived from standard segmentation: left anterior descending, left circumflex, and right coronary artery. Minimal regional values, representing the minimal average values among these 3 vascular territories, where used as representative values for regional MFR (minimum regional MFR), regional stress MBF (minimum regional stress MBF), and regional MFC radius (minimum regional MFC radius).

This methodology is different from the pixel-wise assessment of quantitative flow metrics created by Gould et al. Our methodology is more easily applicable, as pixel-wise assessments are not widely available. The used global and regional average MFR and stress MBF values in the current study are available in most used software solutions. MFC radius could be easily computed, integrating combinations of stress MBF and MFR as per patient single continuous variables. Moreover, global MFR and stress MBF used in the current study are well-studied quantitative parameters since more than two decades. 


\section{Clinical follow-up}

The endpoint of the study was major adverse cardiovascular event (MACE), defined as myocardial infarction, delayed revascularization ( $>6$ months post-PET/CT), heart transplantation, hospitalization for congestive heart failure or de novo stable angina, and cardiac death. Early revascularizations observed within the first 6 months of post-PET/CT were considered to have been triggered by the myocardial perfusion study and were excluded. Hospitalization for de novo stable angina was defined as angina or chest pain of cardiac origin and requiring further investigations and hospitalization. Death from cardiac cause was defined as death from myocardial infarction, congestive heart failure, valvular heart disease, sudden death, death without a witness or of unknown cause, and cardiac interventional/surgical procedure related. Outcome information were obtained from medical records available in the hospital information system. If unsuccessful, participant follow-up was obtained by a phone call to cardiologists or general practitioners and/or participants. In participants with multiple MACE, only the first one was considered for survival analysis. Outcome data were collected in January-February 2021.

\section{Statistics}

We assessed the distribution of data with the Shapiro-Wilk test. Continuous parametric variables were expressed as mean \pm SD and compared using Student's t-tests. Nonparametric data are presented as median [interquartile range] and compared using the Mann-Whitney $U$ test. The chi-square test or Fisher exact test was used for analysis of categorical variables. Receiver-operating characteristic (ROC) analysis and pairwise comparisons were used to compare areas under the curves.

Because dichotomous variables represent a straightforward way to display in survival curves and odds ratios, the Youden index was used to select an optimal threshold value from the ROC for global and minimum regional MFR, stress MBF, and MFC radius measurements. Kaplan-Meier curves were used to elucidate the survival distributions regarding MACE. Differences in the outcomes of participants were assessed using the log-rank test. A Cox proportional hazard regression with adjustment for potential confounders was performed to determine the predictors of worse outcome. To prevent overfitting of the multivariable Cox proportional hazards models, only cardiovascular risk factors with $p$ values $<0.05$ in univariate Cox proportional regression models were considered in the multivariable models. To show the potential incremental value of a quantitative variable, likelihood ratio chi-square test was used. Cox regression analysis and likelihood ratio chi-square test were also performed with global or minimum regional MFR, stress MBF, and MFC radius considered as continuous variables. Only significant results using both binary and continuous variables were considered statistically significant.

Kaplan-Meier curves were also used to elucidate the survival distributions regarding MACE with participants classified into tertiles based on global and regional MFR, stress MBF and MFC radius.

The statistical analysis was performed using R version 4.0.3 (R Foundation for Statistical Computing, Vienna, Austria). A $p$ value of $<0.05$ was considered statistically significant. 


\section{Results}

\section{Participant's characteristics}

From June 2018 to June 2019, ${ }^{82} \mathrm{Rb}$ SiPM PET/CT was performed in 279 participants. Two studies were excluded because of technical issues (delayed start at infusion). Follow-up was successful in 274 of 277 remaining participants (99\%). 40 participants were censored due to early revascularization (5 CABG and 35 percutaneous coronary intervention, $<6$ months after $\mathrm{PET} / \mathrm{CT}$ ) Baseline characteristics of the remaining study population of 234 participants are given in Table 1. Participants had a high prevalence of known CAD (54\%), with a high burden of cardiovascular risk factors (hypertension: 73\%; current or former tobacco use $45 \%$; dyslipidemia: $68 \%$ ). Preventive therapies were highly prescribed in the overall population: $58 \%$ with aspirin, $62 \%$ with beta-blockers, $58 \%$ with angiotensin-converting enzyme inhibitors, and $66 \%$ with lipid-lowering agents. 
Table 1

Baseline Characteristics of the overall population

\begin{tabular}{|ll|}
\hline $\mathbf{n}$ & 234 \\
\hline Age, years & $72[61-78]$ \\
\hline Men, $\mathrm{n}(\%)$ & $153(65.4 \%)$ \\
\hline Body mass index, kg/m² & $31[28-36]$ \\
\hline Cardiovascular risk factors, $\mathrm{n}(\%)$ & $171(73.1 \%)$ \\
\hline Hypertension & $106(45.3 \%)$ \\
\hline Current or former smoker & $159(67.9 \%)$ \\
\hline Dyslipidemia & $85(36.3 \%)$ \\
\hline Diabetes & $35(15 \%)$ \\
\hline Insulin-dependent diabetes & $126(53.8 \%)$ \\
\hline Known CAD & $100(42.7 \%)$ \\
\hline History of myocardial infarction & \\
\hline Medications, $\mathrm{n}$ (\%) & $135(57.7 \%)$ \\
\hline Aspirin & $144(61.5 \%)$ \\
\hline Beta-blockers & $135(57.7 \%)$ \\
\hline ACE inhibitors & $82(35 \%)$ \\
\hline Diuretics & $24(10.3 \%)$ \\
\hline Nitroglycerine therapy & $154(65.8 \%)$ \\
\hline Lipid-lowering agent & \\
\hline CAD - coronary artery disease; ACE - Angiotensin-converting enzyme & \\
\hline
\end{tabular}

\section{Clinical outcomes}

Over the 652 days [IQR: 559 to 751 days] of follow-up, a total of 47 participants experienced a MACE event (13 nonfatal myocardial infarctions, 5 cardiac deaths (1 patient had myocardial infarction and then cardiac death), 10 cases of delayed revascularization, 19 hospitalizations for congestive heart failure or de novo stable angina).

\section{Comparative analysis}


Participants with MACE had significantly worse global or minimum regional MFR, stress MBF, and MFC radius when compared with participants without (Fig. 1). In contrast, global or minimum regional rest MBF were similar among both groups (Table 2). There was a significantly higher prevalence of known CAD or history of myocardial infarction in patients with MACE as compared to patients without MACE (Table 2). 
Table 2

Comparison of participants with and without major adverse cardiovascular events (MACE) during followup

\begin{tabular}{|c|c|c|c|}
\hline & $\begin{array}{l}\text { MACE } \\
(n=47)\end{array}$ & $\begin{array}{l}\text { No MACE } \\
(n=187)\end{array}$ & p-value \\
\hline Age, years & 73 [68-79] & $71[60-77.5]$ & 0.105 \\
\hline Men, n (\%) & $36(77 \%)$ & $117(63 \%)$ & 0.081 \\
\hline Body mass index $\left(\mathrm{kg} / \mathrm{m}^{2}\right)$ & $32[28.5-35.5]$ & $31[28-36]$ & 0.78 \\
\hline \multicolumn{4}{|l|}{ Cardiovascular risk factors, $\mathrm{n}(\%)$ * } \\
\hline Hypertension & $38(81 \%)$ & $133(71 \%)$ & 0.18 \\
\hline Current or former smoker & $26(55 \%)$ & $80(43 \%)$ & 0.12 \\
\hline Dyslipidemia & $34(72 \%)$ & $125(67 \%)$ & 0.47 \\
\hline Diabetes & $20(43 \%)$ & $65(35 \%)$ & 0.32 \\
\hline Insulin-dependent diabetes & $10(21 \%)$ & $25(13 \%)$ & 0.14 \\
\hline Known CAD & $34(72 \%)$ & $92(49 \%)$ & 0.004 \\
\hline History of myocardial infarction & $28(60 \%)$ & $72(39 \%)$ & 0.01 \\
\hline \multicolumn{4}{|l|}{ Medications, $\mathrm{n}(\%)$ * } \\
\hline Aspirin & $28(60 \%)$ & $107(57 \%)$ & 0.35 \\
\hline Beta-blockers & $33(70 \%)$ & $111(59 \%)$ & 0.15 \\
\hline ACE inhibitors & $32(68 \%)$ & $103(55 \%)$ & 0.15 \\
\hline Diuretics & $27(57 \%)$ & $55(29 \%)$ & 0.0002 \\
\hline Nitroglycerine therapy & $6(13 \%)$ & $18(10 \%)$ & 0.61 \\
\hline Lipid-lowering agent & $32(68 \%)$ & $122(65 \%)$ & 0.71 \\
\hline \multicolumn{4}{|c|}{ PET pharmacological stress agent, $n(\%)$} \\
\hline Adenosine & $40(85 \%)$ & $164(88 \%)$ & 0.56 \\
\hline \multicolumn{4}{|c|}{ Haemodynamics during PET/CT, median [IQR] } \\
\hline Rest-HR, bpm & $71[61-81.5]$ & 69 [61-75.5] & 0.12 \\
\hline
\end{tabular}

CAD - coronary artery disease; ACE - Angiotensin-converting enzyme; HR - heart rate, SBP - systolic blood pressure; DBP - diastolic blood pressure; RPP - rate-pressure product $(\mathrm{HR} \times \mathrm{SBP})$; MFR myocardial flow reserve; MBF - Myocardial blood flow; MFC - myocardial flow capacity

* at the time of the PET scan 


\begin{tabular}{|c|c|c|c|}
\hline & $\begin{array}{l}\text { MACE } \\
(n=47)\end{array}$ & $\begin{array}{l}\text { No MACE } \\
(n=187)\end{array}$ & p-value \\
\hline Stress-HR, bpm & 82 [75-99] & $85[74-94.5]$ & 0.72 \\
\hline Rest-SBP, mmHg & $132[118.5-147.5]$ & $137[120-152]$ & 0.34 \\
\hline Stress-SBP, mmHg & 118 [101-131] & $120[107.5-138]$ & 0.07 \\
\hline Rest-DBP, mmHg & 70 [62-75.5] & $70[62-81]$ & 0.49 \\
\hline Stress-DBP, mmHg & $60[52-63.5]$ & $62[55-71]$ & 0.11 \\
\hline Rest-RPP > 8500 & $29(62 \%)$ & $124(66 \%)$ & 0.60 \\
\hline \multicolumn{4}{|l|}{${ }^{82} \mathrm{Rb}$ quantitative imaging, median [IQR] } \\
\hline Global MFR & $1.75[1.395-2.47]$ & $2.49[1.93-3.1]$ & $<0.0001$ \\
\hline Global rest $\mathrm{MBF}, \mathrm{mL} / \mathrm{min} / \mathrm{g}$ & $0.72[0.51-0.925]$ & $0.75[0.59-0.97]$ & 0.34 \\
\hline Global stress $\mathrm{MBF}, \mathrm{mL} / \mathrm{min} / \mathrm{g}$ & $1.5[1.08-1.87]$ & $2.16[1.54-2.88]$ & $<0.0001$ \\
\hline Minimum Regional MFR & $1.56[1.285-2.065]$ & 2.27 [1.655-2.875] & $<0.0001$ \\
\hline Minimum Regional rest $\mathrm{MBF}, \mathrm{mL} / \mathrm{min} / \mathrm{g}$ & $0.81[0.625-1.025]$ & $0.82[0.655-1.06]$ & 0.46 \\
\hline Minimum Regional stress MBF, $\mathrm{mL} / \mathrm{min} / \mathrm{g}$ & $1.22[0.78-1.70]$ & $1.88[1.31-2.52]$ & $<0.0001$ \\
\hline Global MFC radius & $2.30[1.86-3.09]$ & $3.37[2.64-4.24]$ & $<0.0001$ \\
\hline Minimum regional MFC radius & 1.56 [1.285-2.065] & 2.27 [1.655-2.875] & $<0.0001$ \\
\hline \multicolumn{4}{|c|}{$\begin{array}{l}\text { CAD - coronary artery disease; ACE - Angiotensin-converting enzyme; HR - heart rate, SBP - systolic } \\
\text { blood pressure; DBP - diastolic blood pressure; RPP - rate-pressure product (HR } \times \text { SBP); MFR - } \\
\text { myocardial flow reserve; MBF - Myocardial blood flow; MFC - myocardial flow capacity }\end{array}$} \\
\hline * at the time of the PET scan & & & \\
\hline
\end{tabular}

\section{ROC analysis}

On ROC analysis, using the Youden index, we derived the maximum potential effectiveness of global or minimal regional MFR, stress MBF, and MFC radius cutoffs for MACE prediction. For global absolute myocardial perfusion measurements, a threshold of 1.98 for MFR achieved a specificity and sensitivity of $73 \%$ and $64 \%$, a threshold of $1.94 \mathrm{~mL} / \mathrm{g} / \mathrm{min}$ for stress MBF achieved a specificity and sensitivity of $59 \%$ and $83 \%$, and a threshold of 3.12 for MFC radius achieved a specificity and sensitivity of $60 \%$ and $77 \%$. For minimum regional absolute myocardial perfusion measurements, a threshold of 1.75 for MFR achieved a specificity and sensitivity of $72 \%$ and $66 \%$, a threshold of $1.7 \mathrm{~mL} / \mathrm{g} / \mathrm{min}$ for stress MBF achieved a specificity and sensitivity of $61 \%$ and $79 \%$, and a threshold of 2.7 for MFC radius achieved a specificity and sensitivity of $65 \%$ and $77 \%$. Overall, the areas under the ROC curve analyses were 
numerically similar among all such parameters ( $p>0.1$ for all pairwise comparisons), with greater values for global and regional MFC radius $(0.73(0.65-0.80)$ for both; Fig. 2$)$.

\section{Univariate analysis}

The Kaplan-Meier survival curves indicated that participants with global MFR $<1.98$ or stress MBF $<1.94$ $\mathrm{mL} / \mathrm{g} / \mathrm{min}$ or MFC radius $<3.12$ had significantly higher rates of MACE (all $p<0.0001$ ) as compared with those with normal perfusion. Similarly, patients with minimum regional MFR $<1.75$ or stress MBF $<1.7$ $\mathrm{mL} / \mathrm{g} / \mathrm{min}$ or MFC radius $<2.7$ had significantly higher rates of MACE (all $p<0.0001$ ) as compared with those with normal perfusion (Fig. E1 and Fig. E2 in the supplementary data online). Tertiles of global or minimal regional MFR, stress MBF, and MFC radius provided incremental information regarding MACE occurrence (Fig. 3).

On univariable Cox proportional regression, global MFR $<1.98$ or stress MBF $<1.94 \mathrm{~mL} / \mathrm{g} / \mathrm{min}$ or MFC radius $<3.12$, and minimum regional $M F R<1.75$ or stress $M B F<1.7 \mathrm{~mL} / \mathrm{g} / \mathrm{min}$ or $\mathrm{MFC}$ radius $<2.7$ emerged all as significant predictors of MACE (Table 3). Male gender, as well as known history of CAD and history of myocardial infarction were also found to be significantly predictive of MACE (Table 3). When considered as continuous variables, global or minimum regional MFR, stress MBF, and MFC radius also emerged as significant predictors of MACE (Table E1 in the supplementary data online). 
Table 3

Prediction of major adverse cardiovascular events (MACE) during followup. Univariate Cox proportional regression models

\begin{tabular}{|lll|}
\hline & MACE & \\
\cline { 2 - 3 } & Hazard ratio $(95 \% \mathrm{Cl})$ & p-value \\
\hline Global MFR $<1.98$ & $3.68(2.0-6.6)$ & $<0.001$ \\
\hline Global stress MBF $<1.94$ & $5.19(2.6-10.5)$ & $<0.001$ \\
\hline Global MFC radius $<3.12$ & $4.53(2.3-8.9)$ & $<0.001$ \\
\hline Minimum regional MFR $<1.75$ & $3.76(2.1-6.8)$ & $<0.001$ \\
\hline Minimum regional stress MBF $<1.7$ & $4.38(2.3-8.5)$ & $<0.001$ \\
\hline Minimum regional MFC radius < 2.7 & $5.00(2.5-9.8)$ & $<0.001$ \\
\hline Age & $1.02(1.0-1.0)$ & 0.1 \\
\hline Male gender & $2.08(1.1-4.1)$ & $\mathbf{0 . 0 2}$ \\
\hline BMI & $0.99(0.9-1.0)$ & 0.7 \\
\hline Hypertension & $1.77(0.9-3.7)$ & 0.1 \\
\hline Current or former smoker & $1.64(0.9-2.9)$ & 0.09 \\
\hline Dyslipidemia & $1.41(0.7-2.7)$ & 0.3 \\
\hline Diabetes & $1.52(0.9-2.7)$ & 0.2 \\
\hline Known CAD & $2.41(1.3-4.6)$ & $\mathbf{0 . 0 0 5}$ \\
\hline History of myocardial infarction & $2.19(1.2-3.9)$ & $\mathbf{0 . 0 0 8}$ \\
\hline Abbreviations as in Table 2 & & \\
\hline
\end{tabular}

\section{Multivariate Cox regression analysis}

On multivariable analysis after adjustments for sex, known CAD and history of myocardial infarction, (i) Global: $M F R<1.98$, stress MBF $<1.94 \mathrm{~mL} / \mathrm{g} / \mathrm{min}$, and MFC radius $<3.12$, as well as (ii) Minimum regional: $M F R<1.75$, stress $M B F<1.7 \mathrm{~mL} / \mathrm{g} / \mathrm{min}$, and $\mathrm{MFC}$ radius $<2.7$, emerged as all as independent predictors of MACE (Fig. 4). In contrast, male gender, known CAD, and history of myocardial infarction were predictors of events on univariable but not on multivariable analysis (Fig. 4). Considering global values, adjusted HR ranged from 3.1 (95\% Cl: 1.66 to $5.8 ; p<0.001$ ) for MFR to 4.5 (95\% Cl: 2.09 to 9.7; $p$ $<0.001)$ for stress MBF. Considering minimum regional values, adjusted $\mathrm{HR}$ ranged from $3.09(95 \% \mathrm{Cl}$ : 1.64 to $5.8 ; p<0.001$ ) for MFR to 4.25 (95\% Cl: 2.04 to 8.8; $p<0.001)$ for MFC radius. Fig. 5 summarizes the main findings of multivariable analysis. Similar results were observed when global or minimum 
regional MFR, stress MBF, and MFC radius were considered as continuous variables (Fig. E3 in the supplementary data online).

\section{Likelihood ratio chi-square test (Table E2 in the supplementary data online)}

As binary variables, considering minimum regional values, we demonstrated an added value of MFC radius over MFR and stress MBF alone ( $p=0.023$ and $p=0.016$ respectively), as well as an added value of MFR over stress MBF alone and of stress MBF over MFR alone ( $p=0.026$ and $p=0.019$ respectively). As binary variables, considering global values, we demonstrated a trend for an added value of MFC radius over MFR and stress MBF alone ( $p=0.061$ and $p=0.087$ respectively), and an added value of MFR over stress MBF alone and of stress MBF over MFR alone ( $p=0.04$ and $p=0.004$ respectively). However, as continuous variables, no test was significant, except for a trend for an added value of minimum regional MFC radius over MFR alone $(p=0.083)$. Finally, integrating regional perfusion with minimum regional MFC, MFR or stress MBF did not increase prognostic performance compared to global perfusion alone (Table E2 in the supplementary data online).

\section{Discussion}

Our results support that global and regional MFR, stress MBF, and MFC radius, as assessed by using the latest advances in ${ }^{82} \mathrm{Rb}$ PET imaging, are powerful predictors of cardiovascular event, outperforming traditional cardiovascular risk factors such as the presence of known CAD or history of myocardial infarction. In a comprehensive analysis, we show similar results for global and regional MFR, stress MBF, and MFC radius as prognostic factors.

Our study has notable strengths. We utilized state-of-the-art ${ }^{82} \mathrm{Rb} \mathrm{PET}$ imaging, using the latest advances in SiPM PET technology, including a Time of Flight of 214 ps and smaller crystals with higher PET resolution, which greatly ameliorate the image quality as compared to conventional PET with photomultiplier tubes (11). We used standardized automated post processing protocols of the dynamic data including dual spillover, global partial-volume recovery and motion corrections, and resting ratepressure-product (RPP) adjustments $(13,14)$. PET perfusion was quantified by automated, purely objective measurements. All scans were performed using the same machine and the same acquisitions protocols. We had a $99 \%$ successful follow-up rate. We also used a novel quantification technique, MFC radius, that allows per patient single continuous variables to stratify MFC, and, therefore, represents a straightforward tool to select an optimal threshold value for MFC.

There is a major interest in using accurate non-invasive parameters to improve patient assessment and risk stratification. In this line, absolute quantification of the myocardial perfusion as assess by PET/CT offers a powerful opportunity to tackle this challenge $(15,16)$. However, the most reliable quantitative variable on ${ }^{82} \mathrm{Rb}$ cardiac PET/CT for predicting MACE is not fully established and is unknown using SiPM 
technology. Our similar results for global and regional MFC radius, MFR, and stress MBF as prognostic factors were unexpected and show some conflicting results with previous studies.

First, the funding that both global reduced MFR and stress MBF are similar as prognostic factors and are not additive have previously shown conflicting results. Regarding the prediction of cardiovascular deaths, Gupta et al. investigated the importance of global stress MBF and MFR (10). In this study, in univariable analyses, patients with concordant impaired stress MBF and MFR yielded the highest risk of cardiovascular mortality, whereas patients with normal stress MBF and MFR depicted the lowest risk. However, in multivariable analysis, those authors reported that the cardiovascular mortality was independently driven by global MFR, irrespective of whether the global stress MBF was impaired or preserved. Similar findings had been reported by Fukushima et al. in a cohort with almost the same number of patients and using the same radiotracer $\left({ }^{82} \mathrm{Rb}\right)$ that in the current study, but with previous PET technology (17). Taqueti et al. demonstrated that the prognostic value of MFR for the occurrence of MACE was independent of the extent and severity of coronary lesions as evaluated on coronary angiography, but stress MBF was not included in multivariable analysis (18). In an observational study by Patel et al., a threshold of 1.8 for global MFR has been identified to yield a benefit of coronary revascularization over medical treatment, independently of the art of revascularization or the extent of myocardial ischemia at the semi-quantitative analysis, but, again, stress MBF was not studied (19). In the current study using SiPM PET/CT, a threshold of 1.98 for global MFR was retained as the maximum cutoff for MACE prediction, which was interestingly close to their threshold of 1.8 obtained for a different purpose. Contrary, other studies reported that global stress MBF was independently predictive for events, whereas global MFR was not $(20,21)$.

Regional quantitative parameters as expressed by MFC have shown promising clinical data. Gould et al. showed in recent observational studies with large cohorts over long-term follow-up that the extent of severe regional impairment of MFC, expressed as percentage of left ventricular per-pixel regional MFC, provide optimal risk stratification and is associated with a survival benefit gained by revascularization (8, 9). This risk stratification was not entirely assessed by global MFR and global stress MBF (9). Even limited extended area of abnormal regional MFC ( $<0.5 \%$ of the left ventricle) were shown to be predictive of MACE, when integrating the relative stress perfusion (9). Using severe regional MFC impairment as target for revascularization, Bober et al. shown a significant increase of stress MBF was obtained in myocardial territories with impaired MFC, whereas there was no increase in stress MBF in territories with normal MFC (22). Recently, Miura et al. found in a retrospective study that impaired regional modified MFC (utilizing average MFR and stress MBF values for each coronary territory) was associated with both cardiovascular death and MACE, but no comparison was done with traditional quantitative parameters such as stress MBF or MFR (23). In the current study we used a novel quantification technique, MFC radius, that allows per patient single continuous variables to stratify MFC, and, therefore, allows us to select an optimal threshold value for MFC. Using this new tool with a new PET technology, we found that both global and regional MFC values are powerful predictors of cardiovascular event, independent from traditional cardiovascular risk factors, but being similar to global and regional stress MBF or MFR. 
Considering regional stress MBF and MFR, our results are consistent with Bom et al. who found in a $\left[{ }^{15} \mathrm{O}\right]$ water PET study that both global and regional stress MBF had prognostic value in predicting cardiac events, while that the combination of global and regional stress MBF did not improve the prognostic performance compared to either alone (20). Harjulahti et al. found similar results using also [ $\left.{ }^{15} \mathrm{O}\right]$ water PET (4). In contrast, Von Felten et al. recently demonstrated using ${ }^{13} \mathrm{~N}$-ammonia, an independent prognostic value of regional MFR $<2$, being superior to global stress MBF and MFR (24). In this study the authors emphasized several methodological differences for regional measurements between them: (i) a decrease in at least two adjacent segments, (ii) Bom et al.: continuous variables computed as the average MFR values from the two adjacent segments with the lowest values, and (iii) Harjulahti et al.: a single segment with stress MBF lower than $2.3 \mathrm{ml} / \mathrm{g} / \mathrm{min}$. In the current study, the first using the latest SiPM technology, regional absolute perfusion measurements as defined as minimal average values among the 3 vascular territories were not significant predictors of worse outcome as compared to global absolute perfusion measurements. Reduced global or regional minimum MFC radius, MFR or stress MBF were associated with similar risks of MACE after adjustments of different parameters such as previous history of $\mathrm{MI}$ or known CAD. Our results suggest that all these variables have similar significance for the prediction of cardiovascular events and could be applied in a similar way for risk stratification or to potentially guide the risk-based clinical decision to defer for coronary angiography.

\section{Limitations}

This study must be interpreted in the context of its single-center design, with a relatively modest sample size, which limits extensive subgroup analysis for outcomes. The period of follow-up was middle range, with a low incidence of hard cardiac events such as cardiac death. MFC, MFR, and stress MBF have, by definition, interactions. Therefore, these parameters could not be assessed as independent predictors between them without collinearity. Our study emphasized myocardial blood flow measurements, and left ventricular ejection fraction, left ventricular volumes, regional wall motion, coronary artery calcium score and semi-quantitative evaluation of relative perfusion defects were not assessed in this study despite representing important information that could be part of routine PET/CT imaging.

\section{Conclusions}

Using the latest SiPM PET technology with ${ }^{82} \mathrm{Rb}$, global and regional MFR, stress MBF, and MFC radius, are similar powerful predictors of cardiovascular event.

\section{Declarations}

\section{Ethics approval}

All procedures performed in this study were in accordance with the ethical standards of the institutional and/or national research committee and with the 1964 Helsinki declaration and its last amendments or 
comparable ethical standards. The Local Ethics Committee approved this study protocol (\#PB_201700634), and all participants gave written informed consent prior to inclusion.

\section{Consent for publication}

Informed consent was obtained from all individual participants included in the study.

\section{Conflicts of interest/Competing interests}

The authors declare that they have no conflict of interest.

\section{Funding}

Dr. Dietz is supported by Research Fellowship Awards from the Société Française de Radiologie, Paris, France, and from the Agence Régionale de Santé Auvergne-Rhone-Alpes, Lyon, France.

\section{Availability of data and material}

The datasets used and/or analyzed during the current study are available from the corresponding author on reasonable request.

\section{Authors' contributions}

MD, CHK and JOP designed the study. CHK and JOP supervised the study. MD, CHK, GA, VR, SF, MNL, NS, $\mathrm{EE}, \mathrm{OM}$ and JOP contributed to acquisition of clinical and imaging data. MD performed statistical analysis and drafted the manuscript. CHK, GA, VR, SF, MNL, NS, EE, OM and JOP contributed to critical revision of the report. All authors approved the final draft of the manuscript.

\section{Acknowledgements}

The authors would like to thank Pierre Louis Dietz (MSc, Squarepoint Capital, Paris, France) for his scientific help in this study and Christine Geldhof (Lausanne University Hospital, Lausanne, Switzerland) for her help with the Ethics Committee.

\section{References}

1. Knuuti J, Wijns W, Saraste A, et al. 2019 ESC Guidelines for the diagnosis and management of chronic coronary syndromes. Eur Heart J 2020;4:407-77.

2. Juárez-Orozco LE, Tio RA, Alexanderson E, et al. Quantitative myocardial perfusion evaluation with positron emission tomography and the risk of cardiovascular events in patients with coronary artery disease: a systematic review of prognostic studies. Eur Heart J Cardiovasc Imaging 2018;19:117987.

3. Zampella E, Acampa W, Assante R, et al. Combined evaluation of regional coronary artery calcium and myocardial perfusion by 82Rb PET/CT in predicting lesion-related outcome. Eur J Nucl Med Mol 
Imaging 2020 Jul;47:1698-1704.

4. Harjulahti E, Maaniitty T, Nammas W, et al. Global and segmental absolute stress myocardial blood flow in prediction of cardiac events: $\left[{ }^{15} \mathrm{O}\right]$ water positron emission tomography study. Eur $\mathrm{J}$ Nucl Med Mol Imaging 2020 Nov 11 [E-pub ahead of print], https://doi.org/ 10.1007/s00259-020-05093-2.

5. Weber BN, Stevens E, Perez-Chada LM, et al. Impaired Coronary Vasodilator Reserve and Adverse Prognosis in Patients With Systemic Inflammatory Disorders. JACC Cardiovasc Imaging 2021 Mar 10 [E-pub ahead of print], https://doi.org/10.1016/j.jcmg.2020.12.031.

6. Gould KL, Johnson NP, Bateman TM, et al. Anatomic versus physiologic assessment of coronary artery disease. Role of coronary flow reserve, fractional flow reserve, and positron emission tomography imaging in revascularization decision-making. J Am Coll Cardiol 2013;62:1639-53.

7. Johnson NP, Gould KL. Integrating noninvasive absolute flow, coronary flow reserve, and ischemic thresholds into a comprehensive map of physiological severity. JACC Cardiovasc Imaging 2012;5:430-40.

8. Gould KL, Johnson NP, Roby AE, et al. Regional, Artery-Specific Thresholds of Quantitative Myocardial Perfusion by PET Associated with Reduced Myocardial Infarction and Death After Revascularization in Stable Coronary Artery Disease. J Nucl Med 2019;60:410-417.

9. Gould KL, Kitkungvan D, Johnson NP, et al. Mortality Prediction by Quantitative PET Perfusion Expressed as Myocardial flow capacity With and Without Revascularization. JACC Cardiovasc Imaging 2021;14:1020-1034.

10. Gupta A, Taqueti VR, van de Hoef TP, et al. Integrated non-invasive physiological assessment of coronary circulatory function and impact on cardiovascular mortality in patients with stable coronary artery disease. Circulation 2017;136:2325-2336.

11. Koenders SS, van Dalen JA, Jager PL, et al. Value of SiPM PET in myocardial perfusion imaging using Rubidium-82. J Nucl Cardiol 2020 May 14 [E-pub ahead of print], https://doi.org/10.1007/s12350-020-02141-0.

12. Dekemp RA, Declerck J, Klein R, et al. Multisoftware reproducibility study of stress and rest myocardial blood flow assessed with 3D dynamic PET/CT and a 1-tissue-compartment model of 82Rb kinetics. J Nucl Med 2013;54:571-577.

13. Efseaff M, Klein R, Ziadi MC. Short-term repeatability of resting myocardial blood flow measurements using rubidium-82 PET imaging. J Nucl Cardiol 2012;19:997-1006.

14. Hunter CR, Klein R, Beanlands RS, et al. Patient motion effects on the quantification of regional myocardial blood flow with dynamic PET imaging. Med Phys 2016;43:1829.

15. Knuuti J, Ballo H, Juarez-Orozco LE, et al. The performance of non-invasive tests to rule-in and ruleout significant coronary artery stenosis in patients with stable angina: a meta-analysis focused on post-test disease probability. Eur Heart J 2018;39:3322-3330.

16. Driessen RS, Danad I, Stuijfzand WJ, et al. Comparison of Coronary Computed Tomography Angiography, Fractional Flow Reserve, and Perfusion Imaging for Ischemia Diagnosis. J Am Coll Cardiol 2019;73:161-173 
17. Fukushima K, Javadi MS, Higuchi T, et al. Prediction of short-term cardiovascular events using quantification of global myocardial flow reserve in patients referred for clinical 82Rb PET perfusion imaging. J Nucl Med 2011;52:726-32.

18. Taqueti VR, Hachamovitch R, Murthy VL, et al. Global coronary flow reserve is associated with adverse cardiovascular events independently of luminal angiographic severity and modifies the effect of early revascularization. Circulation 2015;6;131:19-27.

19. Patel KK, Spertus JA, Chan PS, et al. Myocardial blood flow reserve assessed by positron emission tomography myocardial perfusion imaging identifies patients with a survival benefit from early revascularization. Eur Heart J 2020;41:759-68.

20. Bom MJ, van Diemen PA, Driessen RS, et al. Prognostic value of [150]H2O positron emission tomography-derived global and regional myocardial perfusion. Eur Heart J Cardiovasc Imaging 2020;21:777-786.

21. Farhad H, Dunet V, Bachelard K, et al. Added prognostic value of myocardial blood flow quantitation in rubidium-82 positron emission tomography imaging. Eur Heart J Cardiovasc Imaging 2013;14:1203-10.

22. Bober RM, Milani RV, Oktay AA, et al. The impact of revascularization on myocardial blood flow as assessed by positron emission tomography. Eur J Nucl Med Mol Imaging 2019;46;1226-1239.

23. Miura S, Naya M, Kumamaru H, et al. Prognostic value of modified coronary flow capacity by $13 \mathrm{~N}$ ammonia myocardial perfusion positron emission tomography in patients without obstructive coronary arteries. J Cardiol 2021. Sept 15 [E-pub ahead of print], https://doi.org/ 10.1016/j.jjcc.2021.09.001.

24. Von Felten E, Benz DC, Benetos G, et al. Prognostic value of regional myocardial flow reserve derived from $13 \mathrm{~N}$-ammonia positron emission tomography in patients with suspected coronary artery disease. Eur J Nucl Med Mol Imaging 2021 Jun 30 [E-pub ahead of print], https://doi: 10.1007/s00259-021-05459-0.

\section{Figures}



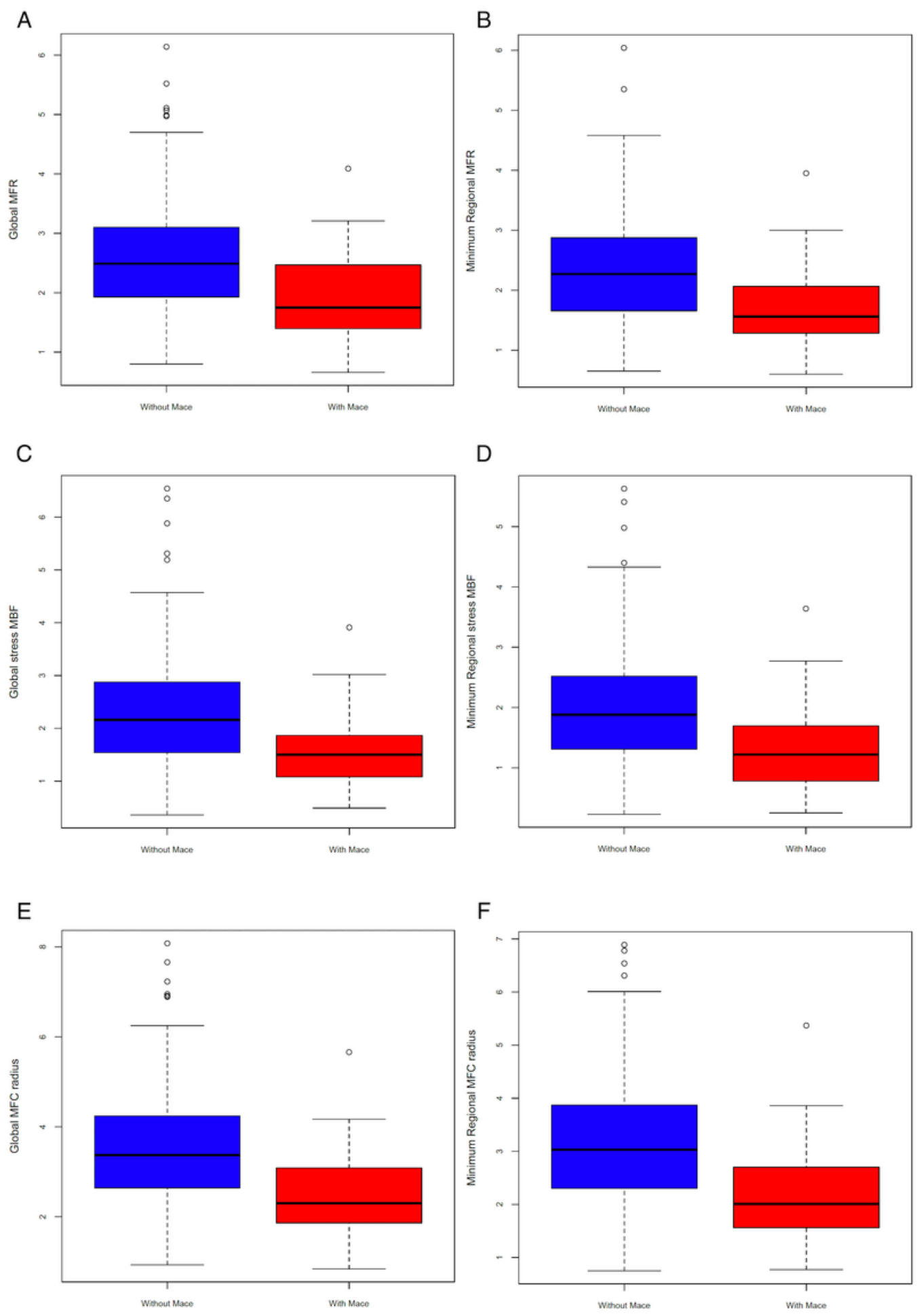

\section{Figure 1}

(A) Global MFR, (B) Minimum regional MFR, (C) Global stress MBF, (D) Minimum regional stress MBF, (E) Global MFC radius, (F) Minimum regional MFC radius, in patients with and without MACE during followup 
A

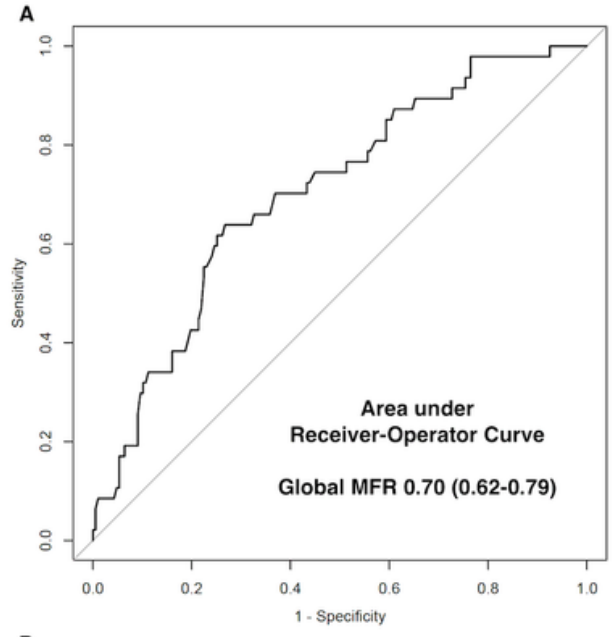

D

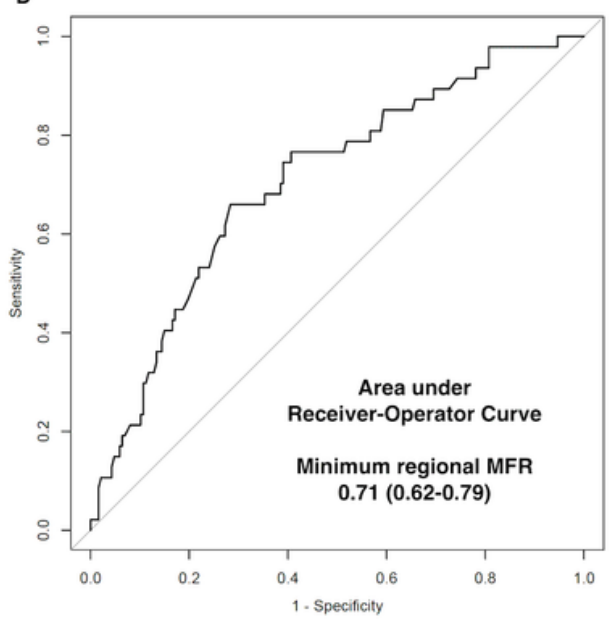

B

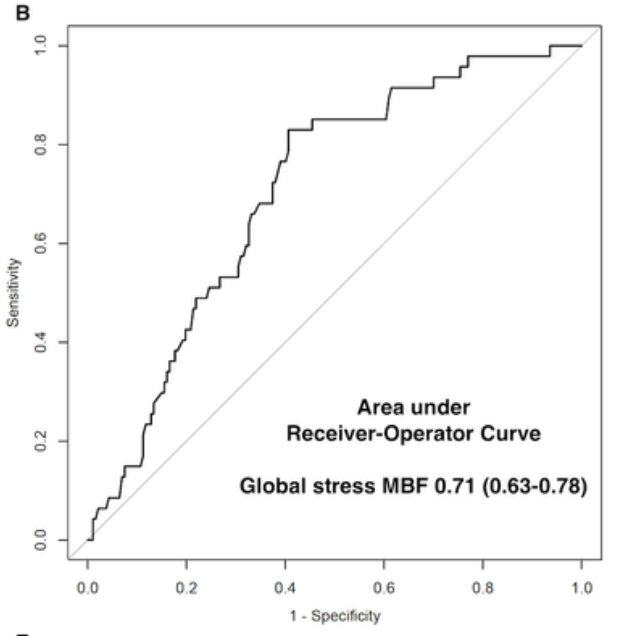

E

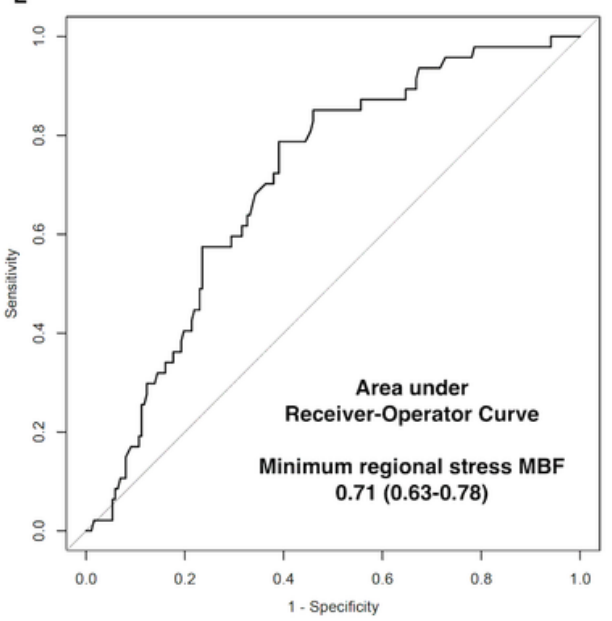

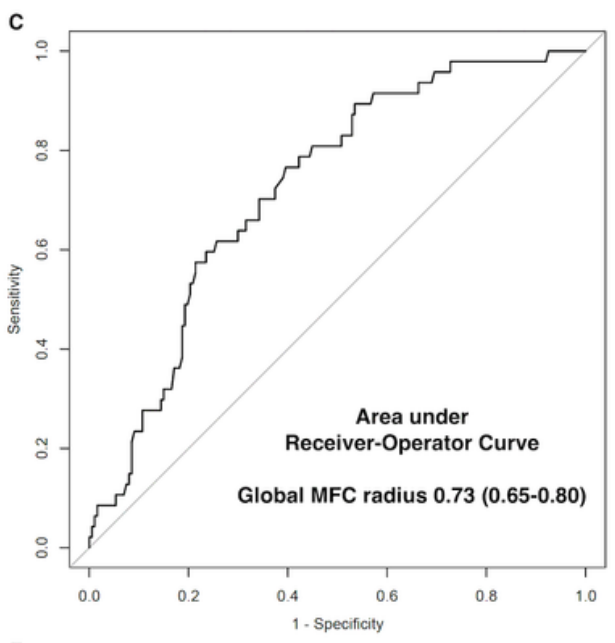

F

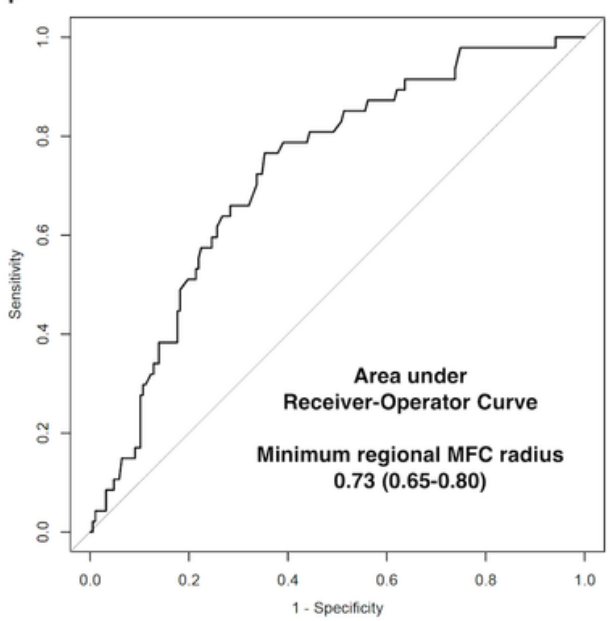

Figure 2

Receiver Operator Curve Analysis for the prediction of MACE in the study population. Receiver operator curves for MACE prediction by global MFR (A), global stress MBF (B), global MFC radius (C), minimum regional MFR (D), minimum regional stress $M B F(E)$, and minimum regional MFC radius $(F)$ 
A

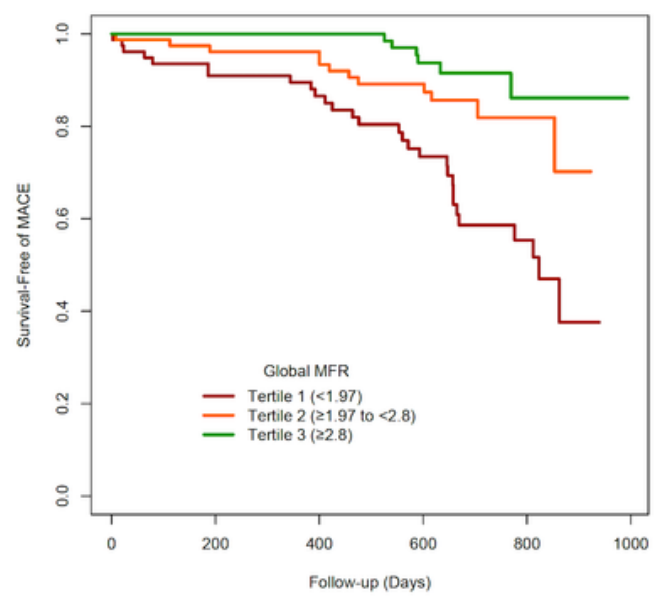

C

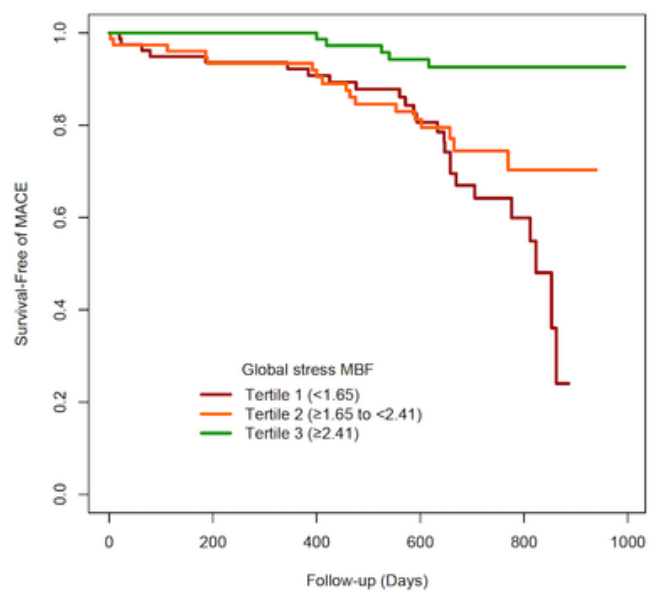

E

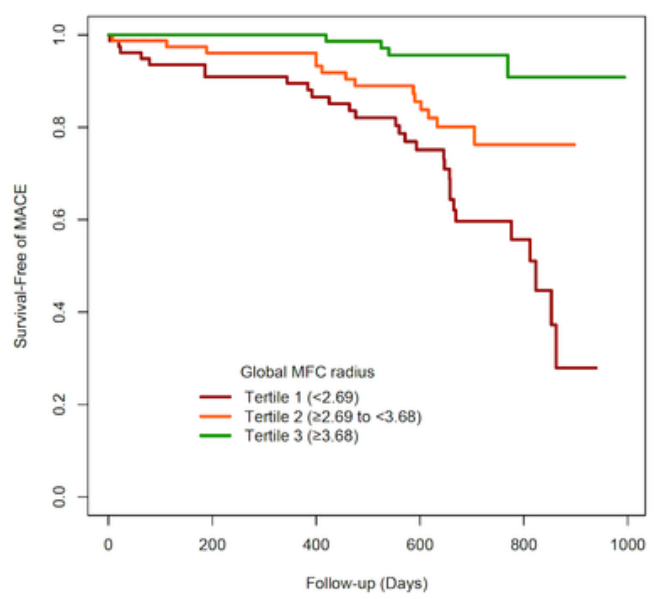

B

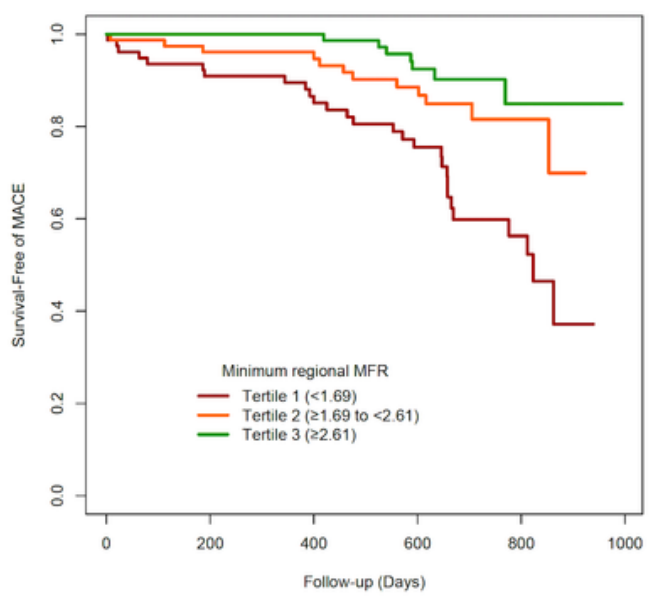

D

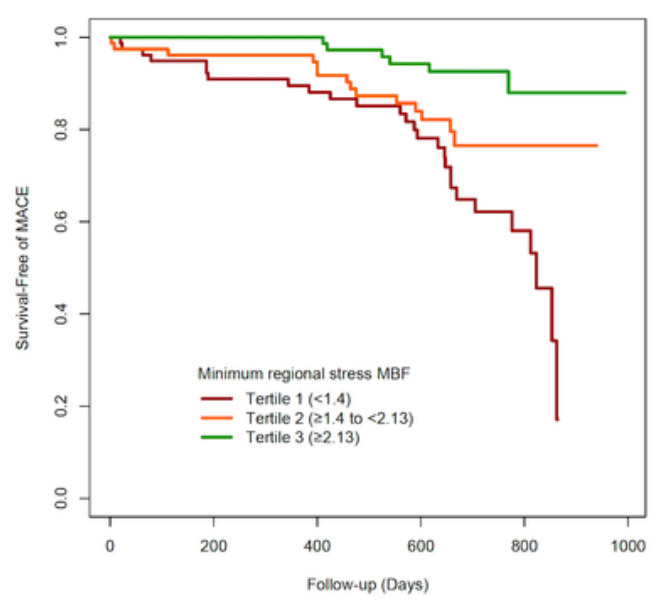

F

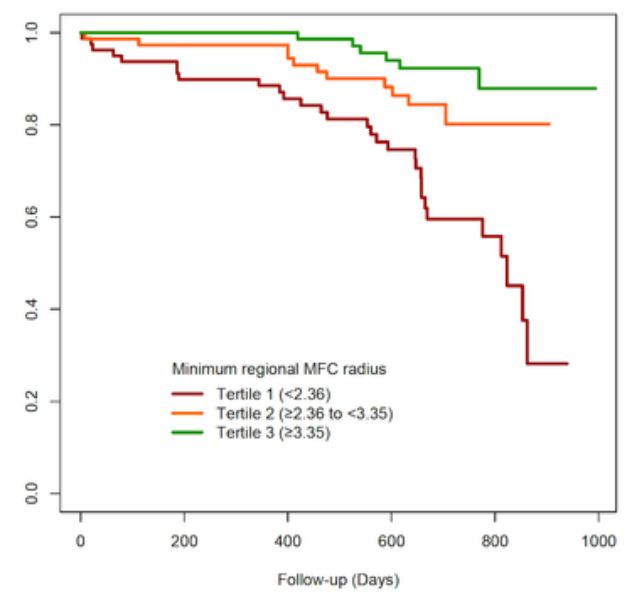

\section{Figure 3}

MACE-free Survival Curves ( $n=234$ ) According to tertiles based on Global and Regional MFR, stress MBF, and MFC radius. (A) Global MFR, (B) Minimum regional MFR, (C) Global stress MBF, (D) Minimum regional stress $M B F,(E)$ Global MFC radius, (F) Minimum regional MFC radius 


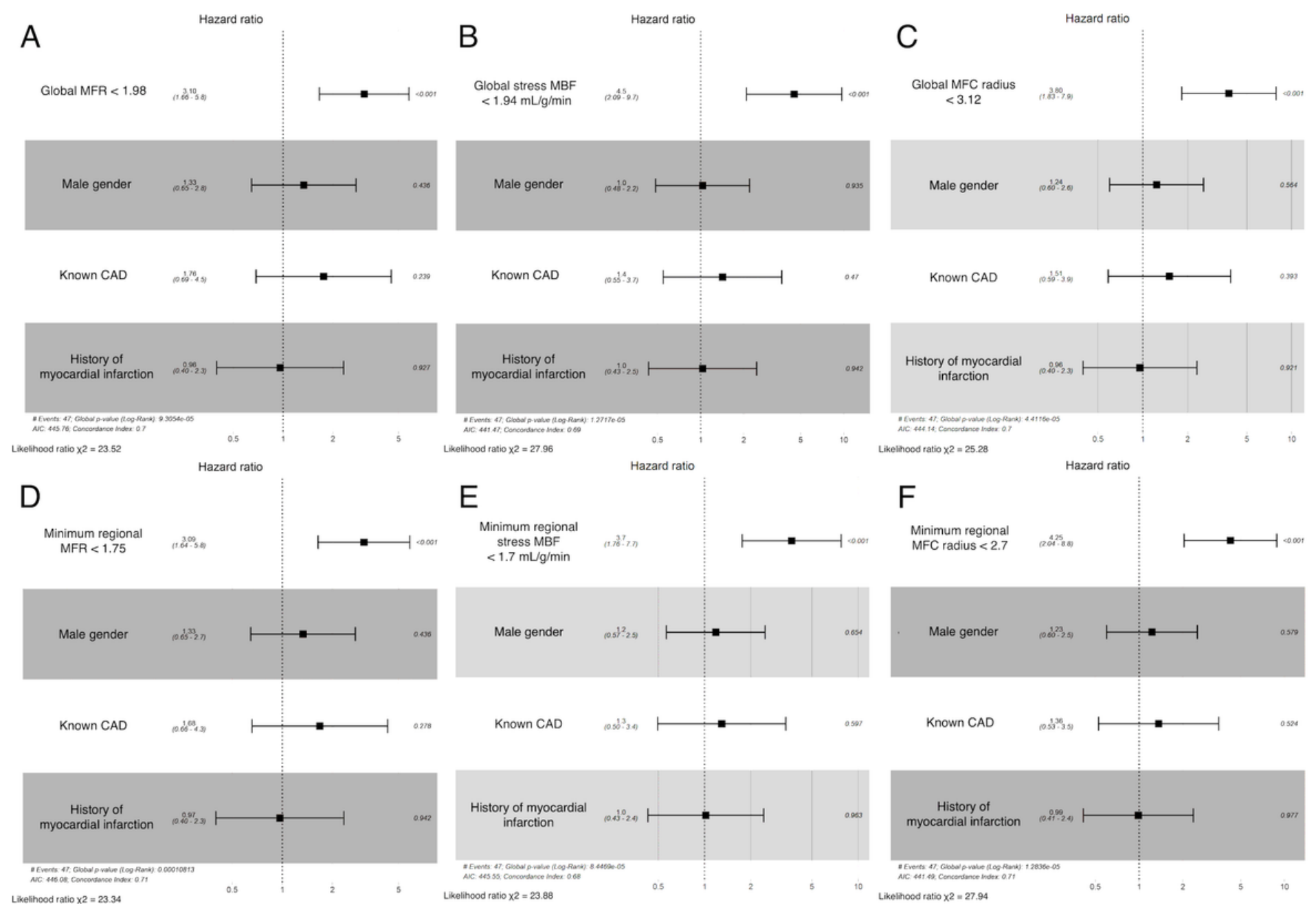

\section{Figure 4}

Predictors of MACE on Cox Proportional Hazards Modeling. Forest plots of hazard ratios derived from multivariable modeling with $95 \%$ confidence intervals for global MFR (A), global stress MBF (B), global $M F C$ radius (C), minimum regional MFR (D), minimum regional stress $M B F(E)$, and minimum regional MFC radius $(F)$ values, along with covariates: male gender, known CAD and history of myocardial infarction 

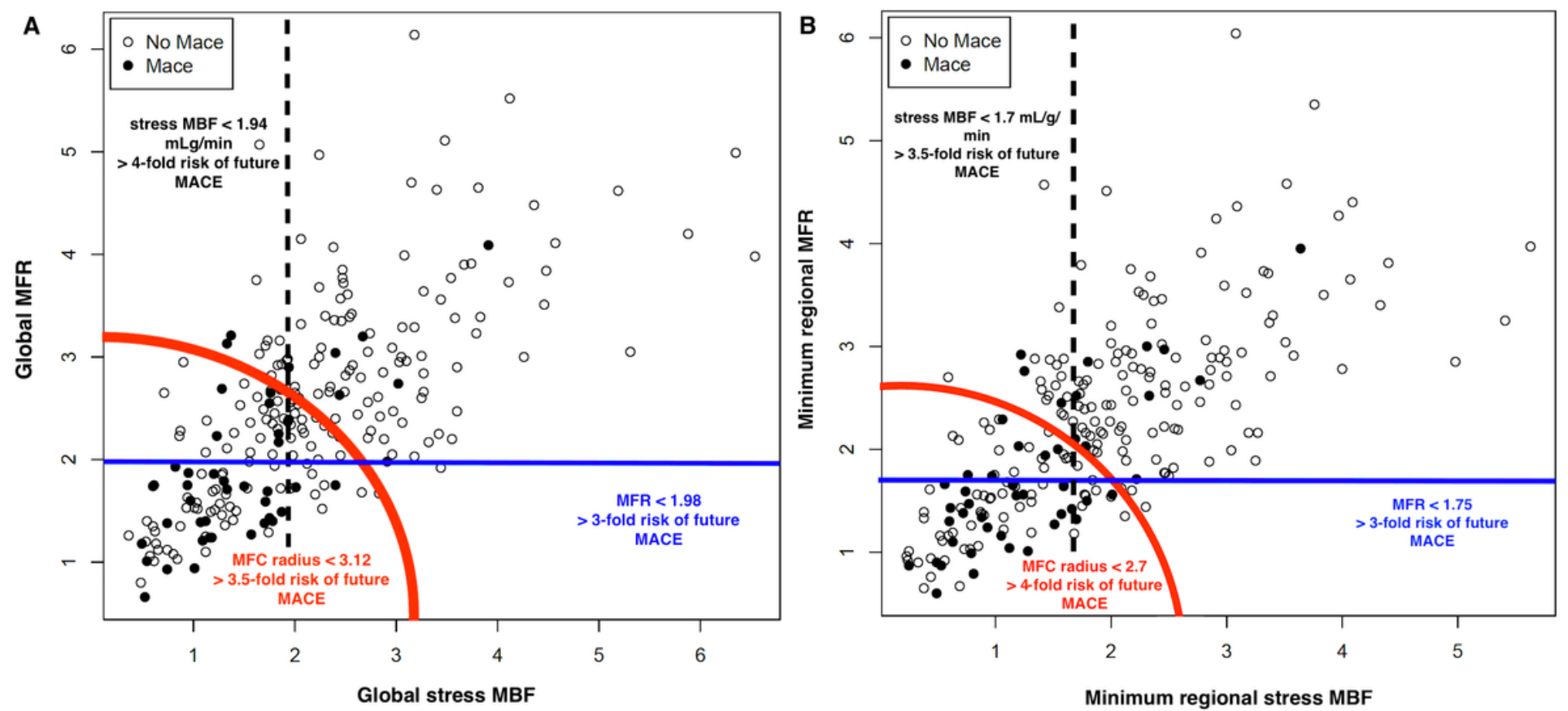

Figure 5

Scatterplots summarizing hazard ratios derived from multivariable analysis for global (A) and minimum regional (B) MFR, stress MBF and MFC radius measurements

\section{Supplementary Files}

This is a list of supplementary files associated with this preprint. Click to download.

- OnlineTablesandOnlineFigures.docx 